\title{
Nanoscale Crystallographic Analysis in FE-SEM Using Transmission Kikuchi Diffraction
}

\author{
Natasha Erdman ${ }^{1}$, Masateru Shibata ${ }^{1}$, Tara Nylese ${ }^{2}$ and Travis Rampton ${ }^{2}$. \\ 1. JEOL USA Inc., 11 Dearborn Rd, Peabody, MA 01960 \\ 2. EDAX Inc., 91 McKee Drive, Mahwah, NJ 07430
}

Recent developments in SEM column design have led to the ability to produce nm spot sizes even at high probe currents [1], thus pushing the analytical techniques available in the SEM to conduct microanalysis with nanometer resolution. Although the limitations of microanalysis at these spatial resolution requirements stem from the physics of beam-specimen interaction and the volume from which the signal is generated during basic bulk sample observation and microanalysis, use of very thin specimens, similar to TEM, can lead to significant improvements in microanalysis resolution. This approach has been shown to be successful in for EDS analysis [2] and has been gaining prominence for crystallographic analysis using Transmission Kikuchi Diffraction (TKD, also referred to as t-EBSD) with a traditional EBSD camera [3].

In this paper we will discuss crystallographic data obtained in FE-SEM with TKD on several different specimen types, in particular thin evaporated metal films and thin films for solar applications. The instrumental setup used in these experiments included a JEOL JSM-7100FT FE-SEM equipped with EDAX Hikari XP camera. The JSM-7100FT is equipped with Aperture angle control lens that allows the user to maintain a small probe size even at large probe currents required for EBSD analysis. The Hikari XP is a standard EBSD camera that is capable of operating at both traditional reflective EBSD geometry and transmission EBSD geometries. Moreover, we have developed a custom holder for the TKD analysis (Fig. 1) where the sample can be tilted up to $-20 \mathrm{deg}$ with respect to the electron beam while the pattern is projected from the underside of the sample onto the EBSD camera phosphor screen. The hybrid lens design of the JSM-7100FT allows EBSD collection even at very short working distances without pattern distortion. In our experiments we have been able to achieve sub $10 \mathrm{~nm}$ resolution on an evaporated $\mathrm{Au}$ film using $30 \mathrm{kV}, 5 \mathrm{nA}$ with the sample positioned at a working distance of $4 \mathrm{~mm}$ (Fig. 2). We have also established that the best pattern quality can be achieved in our setup when the TEM specimen is tilted -20 deg with respect to the electron beam.

Our experience indicates that TKD has a potential to greatly enhance nanocrystalline analysis in SEM, provided that sufficiently thin specimens can be obtained.

\section{References:}

[1] N. Erdman et al., Adv. Mat. Proc., Sept. (2009), p.28-31

[2] N. Rowlands et al., Microsc. Microanal. 15 (2009), p.548-549

[3] P.W. Trimby et al., Acta Mat. 62 (2014), p.69-80 

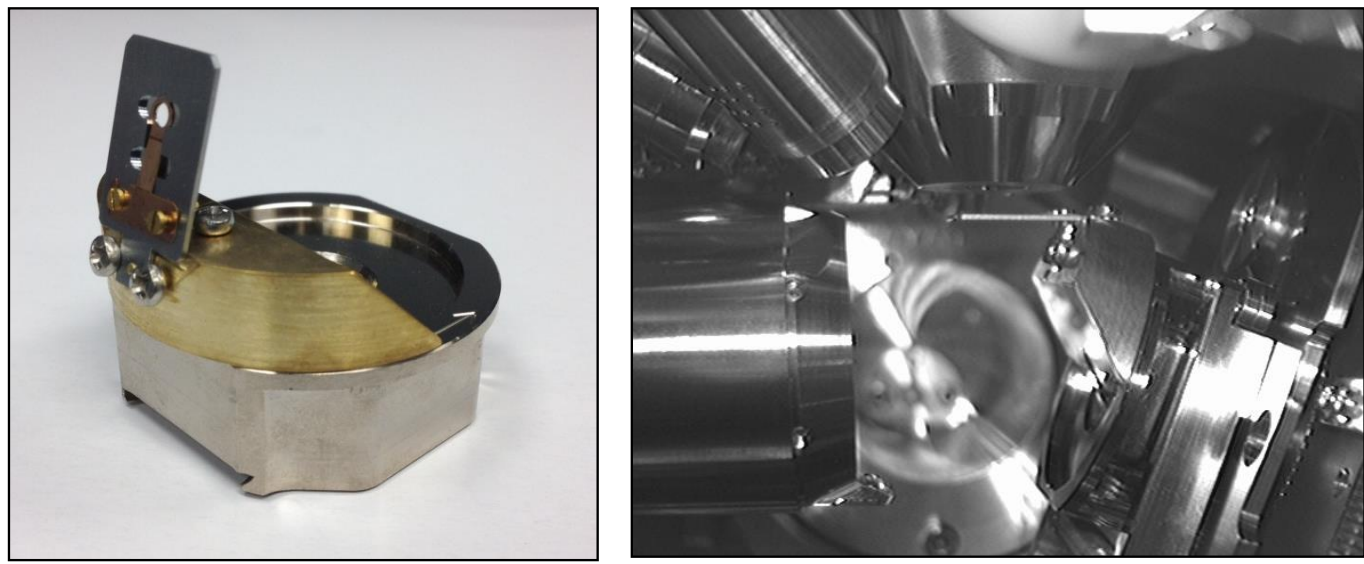

Figure 1. TKD holder (left) and TKD setup in JSM-7100FT (right) with EBSD camera inserted.

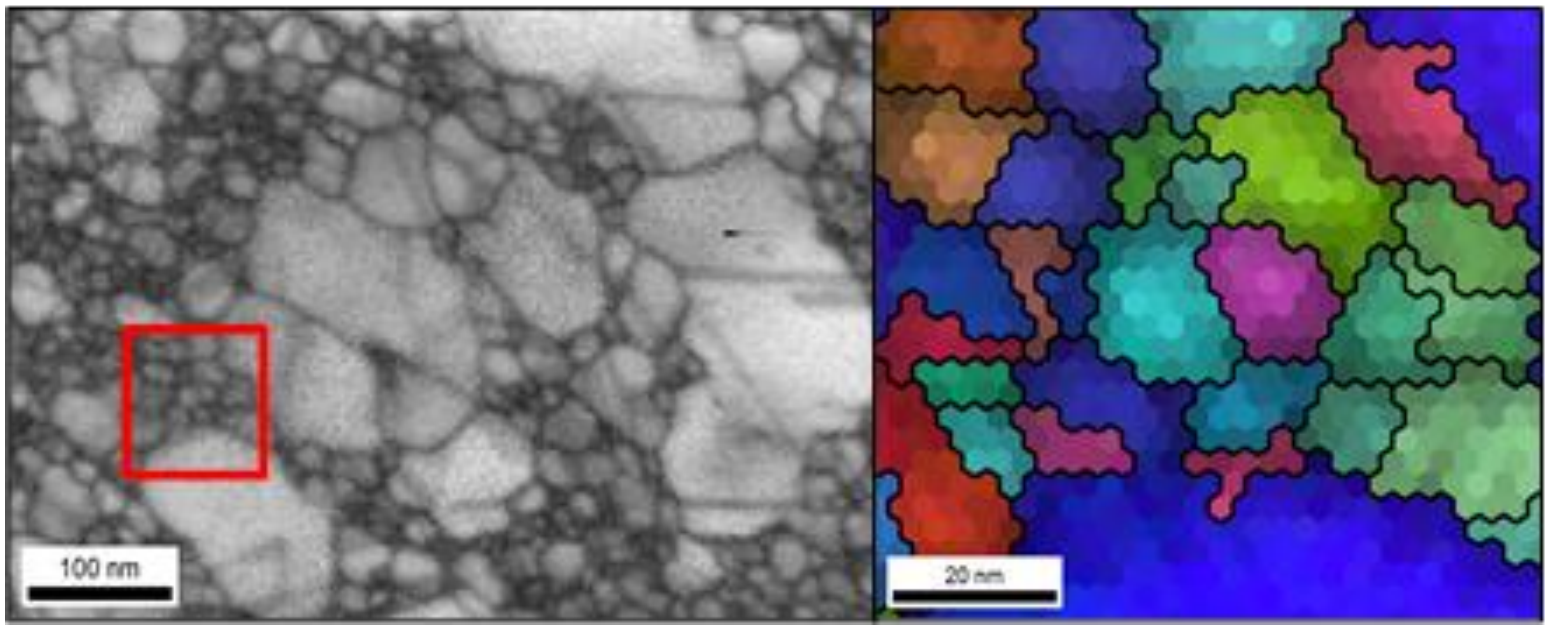

Figure 2. Image quality map and corresponding IPF map (ND) from evaporated Au film analyzed in TKD configuration. Red square shows the analysis area on the right. 